\title{
Spin Force on Pseudo Spin Current in Graphene
}

\author{
S. Zarrin ${ }^{1}$, G. R. Boroun ${ }^{2, *}$ \\ ${ }^{1}$ Physics Department, Razi University, Kermanshah, Iran \\ ${ }^{2}$ Physics Department, Razi University, Kermanshah 67149, Iran \\ *Corresponding Author: boroun@razi.ac.ir
}

Copyright (C)2014 Horizon Research Publishing All rights reserved.

\begin{abstract}
The present study investigated spin force by using pseudo spin-orbit coupling in an artificial atomic structure for graphene. This spin force, which is applied on pseudo spin current, can be controlled by graphene gap and electric field due to charged nanodot. It seems that, by this force can be constructed a logical system from graphene and semiconductors. In addition, this force can be used for understanding the Zitterbewegung of electron wave packet with pseudo spin-orbit coupling in graphene and create of the charge Hall effect in semiconductors and graphene.
\end{abstract}

Keywords Pseudo Spin-Orbit, Graphene, Spin Force, Zitterbewegung

PACS: 85.75.-d, 61.48.Gh

\section{Introduction}

Recently, graphene has developed to one of clearly most active directions of work in current both experimental and theoretical condensed matter physics [1, 2, 3]. As graphene is a two dimensional monoatomic layer of graphite and also it has special honeycomb lattice structure, the fermions in graphene carbon atom are treated as relativistic. The unusual band structure of a graphene is well known [4]. Semenoff, Di Vincenzo and Mele stated that massless excitation states are governed by a wave equation, the Dirac equation, of relativistic quantum mechanics [5, 6]. One important effect which can achieve in a graphene ring geometry by relativistic formalism of quantum theory, is the pseudo spin-orbit coupling producing in semiconductors and geraphene. It provides a route to manipulate and to control the quantum spin states via external fields $[7,8,9,10]$. In quantum physics, the spin-orbit coupling is any interaction of a particle's spin with its motion, describing interaction between the electron's spin, momentum and the magnetic field. The motion of electron spin with spin-orbit coupling in an electromagnetic field is understandable. By this appeared pseudo spin effect in graphene, a logical system can be constructed. Jalil et al presented a pseudo SO coupling effect in graphene in a proposed ring-and-dot nanostructure. According to that work, charge accumulation at the central graphene ring produces a radial electric field, while particles circulation around the ring analogue to the orbital motion of electrons around the atomic nucleus $[11,12,13,14]$.

In electrodynamics a magnetic field would exert a Lorentz force on a movable electric charge. This Lorentz force can generate the Hall effect in solid. In doped semiconductors the spin orbit (SO) interaction leads to a spin-Hall effect, in which a spin current flows perpendicular to an applied electric field [15, 16, 17]. In the electromagnetic field, the interaction of the spin behaves like a gauge charge and belongs to the SU(2) gauge field. The force originates from the spin-orbit coupling which can be derived from the Schrodinger equation for an electron in an electromagnetic potential. From an exact solution of a single electron with the spin-orbit coupling, it is crucial to understand the Zitterbewegung of the electronic wave packet is driven by spin force on a moving spin [18]. The phenomenon of Zitterbewegung is similar to beating effect in classical wave mechanics with different frequencies. The Zitterbewegung is direct consequence of the coupling of different energy eigenstates for systems with more than one degree of freedom [19]. Also, the Zitterbewegung can be observed in spintronic 
systems as well [20], and is not absolutely a relativistic effect [21, 22, 23, 24, 25, 26] but Gyula David and et al initiated other works with an aim to demonstrate the appearance of the Zitterbewegung not only for relativistic Dirac electrons [19]. The generation of the spin currents in both rotationally and linearly accelerating systems has been investigated theoretically. The explicit form of the spin-dependent force acting on electrons in a rotating frame in the presence of electromagnetic fields was derived from the Dirac equation [27]. It is essential that the electron's spin is an intrinsic quantum variable, not a just classical tiny magnetic moment.

In this work, we use an artificial atomic structure, consisting several rings and a charged nanodot at the center of central ring in this structure. A high pseudo spin-orbit coupling strength can be achieved by accelerating the Dirac particles around the rings [7]. We should notice that this structure grown on $\mathrm{SiC}$ and experiments have shown that the energy gap experienced by Dirac particles in monolayer graphene grown on $\mathrm{SiC}$ is approximately $0.26 \mathrm{eV}$, and can be as low as $0.04 \mathrm{eV}$, with proper engineering [28, 29]. Thus, using external electromagnetic field, it will be really possible to control the some physical parameters of graphene as magnetic moment. Considering this fact that pseudo spin magnetism might exist in graphene [30], we encounter the use of our structure as a memory or logical device which binary states are based on the pseudo spin moment. We can use the pseudo spin-orbit coupling to achieve current-induced switching of this memory. the spin force, which is produced by pseudo spin-orbit coupling is the main factor of this switching. To switch the pseudo spin moment which varies by the spin force, we pass the Dirac particles in the clockwise direction around the ring by applying a voltage. In the following, we drive the spin force, spin current and Zitterbewegung amplitudes which depend on electric field due to charged nanodat at the central graphene ring. Also, the role of spin force in generation of the spin Hall effect which driven by the spin current in graphene has been discussed.

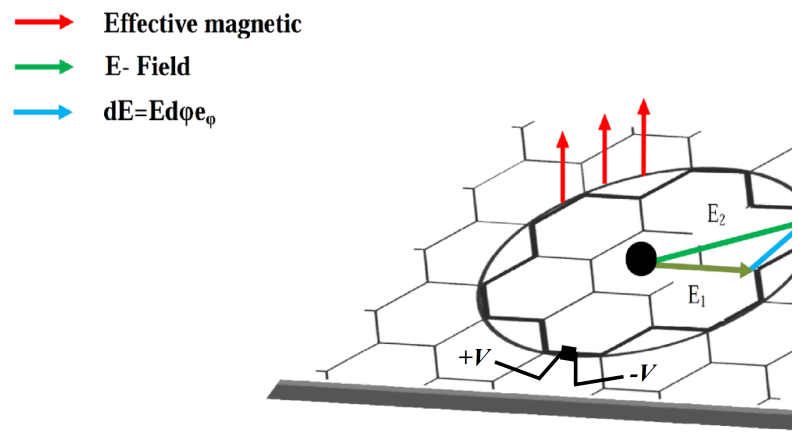

Figure 1. Graphene rings-and-dot nanostructure

\section{Method}

Foldy-Wouthuysen representation can be used for particles subjected in an electromagnetic field. The Foldy-Wouthuysen transformation, as an approximation of the exact solution, is only applicable to weak fields, which improves the approach in a systematic manner. If external fields are coupled to a Hamiltonian $\hat{H}$, then $\hat{H}$ always contains parts coupling together the positive and negative energy solution. In the case of weak fields the role of the odd parts of this operator can be neglected. So, Hamiltonian is:

$$
\hat{H}=\hat{\beta} m_{0} c^{2}+\hat{\varepsilon}+\hat{O}
$$

where $\hat{\beta}$ - is $4 \times 4$ Dirac matric. This Hamiltonian corresponds to be remaining even parte of $\hat{\varepsilon}$ and $\hat{O}$ to the odd part. External electromagnetic field yields:

$$
\hat{O}=c \boldsymbol{\alpha} \cdot\left(\hat{\mathbf{p}}-\frac{e}{c} \mathbf{A}\right), \hat{\varepsilon}=e V(r) .
$$

In which $\hat{\alpha}$ - is $4 \times 4$ Dirac matric and $V$ is scalar potential and $\mathbf{A}$ is a vector potential for magnetic field, in analogy to the case of free fields. The transformation demonstrated below is introduced:

$$
\hat{H}^{\prime}=e^{i \hat{S}} \hat{H} e^{-i \hat{S}}
$$

with the intention of minimizing the odd parts of the Hamiltonian $\hat{H}^{\prime}$, or even to make them vanish.

$$
\hat{S}=\frac{i}{2 m c^{2}} \hat{\beta} \hat{O}
$$


whereas $S$ shall not explicitly depend on time. So we can write Eq. (3) to this form.

$$
\hat{H}^{\prime}=\hat{H}+i[\hat{S}, \hat{H}]+\frac{i^{2}}{2}[\hat{S},[\hat{S}, \hat{H}]]+\frac{i^{3}}{6}[\hat{S},[\hat{S},[\hat{S}, \hat{H}]]]+\cdots
$$

Now we calculate the various commutators of $\hat{S}$ and $\hat{H}$ in the equation above as follows.

$$
\hat{H}^{\prime}=\hat{\beta}\left(m c^{2}+\frac{1}{2 m c^{2}} \hat{O}^{2}-\frac{1}{8 m^{3} c^{6}} \hat{O}^{4}\right)+\hat{\varepsilon}-\frac{1}{8 m^{2} c^{4}}[\hat{O},[\hat{O}, \hat{\varepsilon}]]+\frac{1}{2 m c^{2}} \hat{\beta}[\hat{O}, \hat{\varepsilon}]-\frac{1}{3 m^{2} c^{4}} \hat{O}^{3},
$$

in Eq.(6) we use of the relations $\hat{\beta} \hat{O}=-\hat{O} \hat{\beta}$ and $\hat{\beta} \hat{\varepsilon}=\hat{\varepsilon} \hat{\beta}$, and we can write this equation as follow:

$$
\hat{H}^{\prime}=\hat{\beta} m_{0} c^{2}+\hat{\varepsilon}^{\prime}+\hat{O}^{\prime}
$$

At this step we might simply argue: Let us omit all odd terms of $\hat{H}^{\prime}$ i.e. the last term Eq.(7) with the odd powers of $\hat{O}$. If we perform a second transformation:

$$
\hat{S}^{\prime}=-\frac{i}{2 m c^{2}} \hat{\beta} \hat{O}^{\prime}=-\frac{i}{2 m c^{2}} \hat{\beta}\left(\frac{1}{2 m c^{2}} \hat{\beta}[\hat{O}, \hat{\varepsilon}]-\frac{1}{3 m^{2} c^{4}} \hat{O}^{3}\right)
$$

and then it obtains:

$$
\hat{H}^{\prime \prime}=e^{i \hat{S}^{\prime}} \hat{H}^{\prime} e^{-i \hat{S}^{\prime}}
$$

The term proportional to $O^{\prime 3}$ contains large powers of $\frac{1}{m c^{2}}$ and therefore it can be neglected, thus $\hat{H}^{\prime \prime}$ is given by:

$$
\hat{H}^{\prime \prime}=\hat{\beta} m c^{2}+\hat{\varepsilon}^{\prime}+\frac{1}{2 m c^{2}} \hat{\beta}\left[\hat{O}^{\prime}, \hat{\varepsilon}^{\prime}\right]
$$

To eliminate $O^{\prime \prime}$, we apply a third transformation by neglecting the odd terms proportional to $\frac{1}{m^{2} c^{4}}$ this yield [31]:

$$
\hat{H}^{\prime \prime \prime} \cong \hat{\beta} m c^{2}+\hat{\varepsilon}^{\prime}=\hat{\beta}\left(m c^{2}+\frac{1}{2 m c^{2}} \hat{O}^{2}-\frac{1}{8 m^{3} c^{6}} \hat{O}^{4}\right)+\hat{\varepsilon}-\frac{1}{8 m^{2} c^{4}}[\hat{O},[\hat{O}, \hat{\varepsilon}]] \equiv \hat{H}_{\Phi}=\hat{\beta} E_{p},
$$

Where $E_{p}$ is particles energy. So the Hamiltonian obtain like this,

$$
\begin{aligned}
& \hat{H}_{\Phi} \cong \hat{\beta}\left(m c^{2}+\frac{1}{2 m}\left(p-\frac{e}{c} A\right)^{2}\right)-\frac{e \hbar}{4 m^{2} c^{2}} \boldsymbol{\sigma} \cdot(\mathbf{E} \times \mathbf{p})- \\
& -\frac{e \hbar}{2 m c} \hat{\beta}(\boldsymbol{\sigma} \cdot \mathbf{B})-\frac{i e \hbar^{2}}{8 m^{2} c^{2}} \boldsymbol{\sigma} \cdot(\nabla \times \mathbf{E})+e V-\frac{e \hbar^{2}}{8 m^{2} c^{2}} \nabla \cdot \mathbf{E},
\end{aligned}
$$

where $\sigma=\left(\sigma^{1}, \sigma^{2}, \sigma^{3}\right)$ - are Pauli matrices. If $B=0$, the Hamiltonian become,

$$
\begin{gathered}
i \hbar \frac{\partial}{\partial t} \psi=H_{\Phi} \psi, \psi=\left(\begin{array}{c}
\varphi \\
\chi
\end{array}\right) e^{-\frac{i m c^{2} t}{\hbar}}, \\
H_{\varphi}=\frac{p^{2}}{2 m}+\frac{e \hbar}{8 m^{2} c^{2}} \boldsymbol{\sigma} \cdot(\mathbf{p} \times \mathbf{E})+V_{e f f}-\frac{e \hbar}{8 m^{2} c^{2}} \boldsymbol{\sigma} \cdot(\mathbf{E} \times \mathbf{p}),
\end{gathered}
$$

in which $V_{e f f}=e V-\frac{e \hbar^{2}}{8 m^{2} c^{2}} \nabla \cdot \mathbf{E}, \mathbf{p} \times \mathbf{E}=-i \hbar \nabla \times \mathbf{E}-\mathbf{E} \times \mathbf{p}$. Using Heisenberg equation the kinetic velocity is:

$$
\mathbf{v}=\frac{1}{i \hbar}[\mathbf{r}, H]=\frac{1}{m}\left(\mathbf{p}+\frac{e}{c} \boldsymbol{\Lambda}\right)
$$

where $\boldsymbol{\Lambda}=\frac{\hbar}{4 m c}(\mathbf{E} \times \boldsymbol{\sigma})$ comes from the pseudo spin-orbit coupling. It indicates clearly that $\boldsymbol{\Lambda}$ plays role of a $S U(2)$ gauge vector potential. The spin dependence of the gauge field can splits the charged carriers with different spins in cyclotron motion experimentally [32]. With this velocity, the Hamiltonian become:

$$
H_{\varphi}=\frac{m}{2}\left(v^{2}-\left(\frac{e}{m c}\right)^{2} \Lambda^{2}\right)+V_{e f f}
$$

where $\Lambda^{2}=2\left(\frac{\hbar}{4 m c}\right)^{2}|E|^{2}$, and the spin force is calculated by applying again Heisenberg equation as follows:

$$
\begin{gathered}
f_{1}=\frac{e}{2 c}(\mathbf{v} \times \mathrm{B}-\mathrm{B} \times \mathbf{v})-\nabla V_{e f f}, \\
f_{2}=\frac{\mu_{B e f f}^{2}}{4 m c^{2}}[\nabla(\mathbf{E} \cdot \mathbf{E})-i(\nabla \times \mathbf{E})(\mathbf{E} \cdot \boldsymbol{\sigma})],
\end{gathered}
$$




$$
f_{3}=-\frac{e^{2} \hbar}{16 m^{2} c^{4}}[(\mathbf{E} \cdot \boldsymbol{\sigma})(\mathbf{E} \times \mathbf{v})+(\mathbf{E} \times \mathbf{v})(\mathbf{E} \cdot \boldsymbol{\sigma})] .
$$

Where $\mathrm{B}=\nabla \times \boldsymbol{\Lambda}$ and $\mu_{\text {Beff }}=\frac{e \hbar}{2 m c}$ are gauge magnetic field and pseudo magnetic moment, respectively. An effective magnetic magnetization and permeability for this structure of graphene can be obtained by effective pseudo magnetic moment in non-relativistic limit. The terms, $f_{2}, f_{3}$, are inversely related to the energy gap $\Delta=2 m c^{2}$ between a particle and a hole. Experiments have shown that the energy gap experienced by Dirac particles in monolayer graphene grown on $\mathrm{SiC}$ is approximately $0.26 \mathrm{eV}$, and can be as low as $0.04 \mathrm{eV}$, with proper engineering [28, 29]. So we should notice, the uncertainty relationship tells that the position and momentum cannot be measured simultaneously, and there is no concept of force in quantum mechanics. To see the physical meaning of the equation, we take the expectation values of both sides with respect to a Heisenberg state $|\Phi\rangle$ which does not varies with time. The observable expectation values describe the central motion in the wave package of electrons. The first term $f_{1}$ in Eq.(17) is the Lorentz force for a charged particle in a magnetic field $\langle B\rangle$ which contains the contribution from the $S U(2)$ gauge field $\boldsymbol{\Lambda}$ as well as the conventional electromagnetic field. We have recovered the Ehrenfest theorem as one of the examples of the corresponding principle in quantum mechanics. The term $f_{2}$ comes from the radial electric field (because of $d E=E d \phi \hat{e_{\phi}}$ then $\nabla \times \mathbf{E} \neq 0$ ). The last term, $f_{3}$ comes from the radial electric field and the $S U(2)$ gauge potential or spin-orbital coupling. This force drives electrons to form a charge Hall current perpendicular to the spin current. To see the physical meaning of the force, we write spin force for a single electron on a quantum state in a compact form :

$$
\left\langle f_{3}\right\rangle=-\frac{e^{2}}{\Delta^{2}}\left\langle\left(|E| \mathbf{E} \times \mathbf{J}_{s}^{E}\right)\right\rangle,
$$

where the spin current is defined, $\mathbf{J}_{s}^{E}=\frac{\hbar}{4}\left\{\mathbf{v}, \boldsymbol{\sigma} \cdot \frac{\mathbf{E}}{|E|}\right\}$. This force is related to the square of electric field $E$ and the pseudo spin current which polarization is projected along electric field. For the ring structure of Fig.(1), the magnitude of the electric field is $|E|=\frac{N e}{4 \pi \varepsilon_{0} r^{2}}$, where $\mathrm{r}$ is a ring radius which encompass graphene rings, $N$ is the number of discrete charges in the nanodot and $\varepsilon_{0}=8.85 \times 10^{-12} C^{2} j^{-1} m^{-1}$ is the permittivity of free space. Since the momentum $p, r$ are suitable quantum numbers, without loss of generality, we take $p=p_{x}$ just along the $x$ direction and $E=E_{y}$ just along the $y$ direction. Direction of the pseudo spin current depends on momentum and gauge field direction, if initial spin state would be along with $z$ direction, $\mathbf{J}_{s}^{E}, \mathbf{f}_{3}=0$, but if initial spin state would be within graphene sheet $\mathbf{J}_{s}^{E}, \mathbf{f}_{3} \neq 0$. Therefor the pseudo spin current just exists within graphene sheet and spin force always is along with $z$ direction, Fig.(2). Due to the pseudo spin-orbit

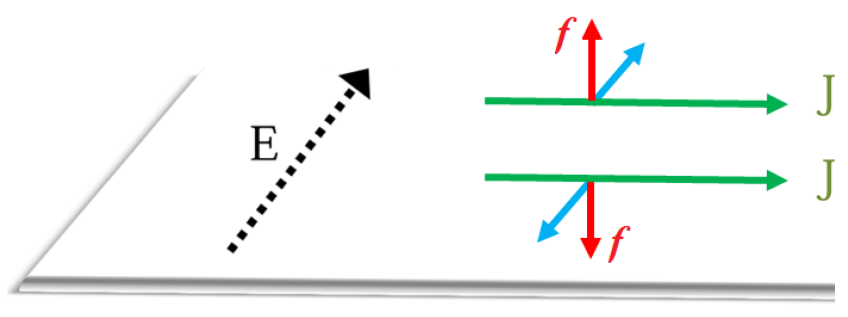

Figure 2. The electric field exerts opposite forces on electrons with different spins polarized along the field.

coupling the electron's spin will precess with time,

$$
\frac{d \sigma}{d t}=\Omega \times \sigma
$$

where $\boldsymbol{\Omega}=\frac{e}{4 m^{2} c^{2}}(\mathbf{p} \times \mathbf{E}-\mathbf{E} \times \mathbf{p})$ is the Larmor frequency. The pseudo spin-orbit coupling provides an effective magnetic field along the $z$ direction, $B_{e f f}=\frac{1}{m c}(\mathbf{p} \times \mathbf{E}-\mathbf{E} \times \mathbf{p})$. The electron's spin precesses in the spin $x-y$ plane $\sigma_{x}(t)=$ $\sigma_{x} \cos (\Omega t)-\sigma_{y} \sin (\Omega t), \sigma_{y}(t)=\sigma_{y} \cos (\Omega t)+\sigma_{x} \sin (\Omega t)$ and $\sigma_{z}(t)=\sigma_{z}$. Therefor, the spin $\sigma_{x}, \sigma_{y}$ vary with time and the spin current is always along the $x$ direction $\mathbf{J}_{s}^{y}=\frac{\hbar}{2 m} p_{x}\left(\sigma_{y} \cos (\Omega t)+\sigma_{x} \sin (\Omega t)\right) \hat{x}$ and also spin force on the spin is perpendicular to the $x$ direction. The kinetic velocities of $v_{x}$ and $v_{z}$ at a time $t$ are:

$$
v_{x}=\frac{p_{x}}{m}+\frac{e \hbar}{4 m^{2} c^{2}} E \sigma_{z} \quad, \quad v_{z}=-\frac{e \hbar}{4 m^{2} c^{2}} E \sigma_{x} .
$$


The velocity along the $z$ direction will form a Hall current orthogonal to the spin current. This is the charge Hall effect driven by the spin current. Integrating of Eq.(20) with respect to time, as we have:

$$
\mathbf{x}(t)-\mathbf{x}(0)=\mathbf{V} t+\frac{E e \hbar}{4 m^{2} c^{2}} \int\left(\sigma_{z} \hat{i}-\sigma_{x} \hat{j}\right) d t,
$$

where

$$
\mathbf{V}=\frac{p_{x}}{M} \hat{i}
$$

so

$$
\langle\mathbf{x}(t)\rangle=\langle\mathbf{x}(0)\rangle+\langle\mathbf{Z}(t)\rangle+\langle\mathbf{V} t\rangle
$$

whereas the so-called $\mathbf{V}$ and $\mathbf{Z}$ are velocities and Zitterbewegung amplitudes respectively [27], although $p_{z}=0$ the kinetic velocity $v_{z}$ oscillates with the frequency $\omega_{1}$ while $v_{x}$ remains constant. The $z$-component of the position is,

$$
\left\langle z_{1}(t)\right\rangle=\left\langle z_{1}(0)\right\rangle-\left\langle\frac{e \hbar E_{y}}{\Delta m \omega_{1}} \sin \left(\frac{\omega_{1} t}{2}\right)\left(\sigma_{x} \cos \left(\frac{\omega_{1} t}{2}\right)-\sigma_{y} \sin \left(\frac{\omega_{1} t}{2}\right)\right)\right\rangle .
$$

But if $p=p_{y}$ and $E=E_{x}$, so the pseudo spin current is along with the $y$ direction and also spin force on the spin is perpendicular to the $y$ direction. Therefore ,the $z$-component of the position is:

$$
\left\langle z_{2}(t)\right\rangle=\left\langle z_{2}(0)\right\rangle-\left\langle\frac{e \hbar E_{x}}{\Delta m \omega_{2}} \sin \left(\frac{\omega_{2} t}{2}\right)\left(\sigma_{y} \cos \left(\frac{\omega_{2} t}{2}\right)+\sigma_{x} \sin \left(\frac{\omega_{2} t}{2}\right)\right)\right\rangle .
$$

Where $\omega_{1}=\left\{p_{x}, E_{y}\right\}$ and $\omega_{2}=\left\{p_{y}, E_{x}\right\}$ are the Larmor frequencies. In the equations (22),(23), the Zitterbewegung component is dependent on the graphene's energy gape and electric field and also these equation haven't velocity component. Because the carriers in graphene rotate around the rings, they have $p_{x}, p_{y}, E_{x}, E_{y}$. The Eq.(22) and Eq.(23) are not equal, the difference of between these positions is $\delta\langle z\rangle=\left\langle z_{2}\right\rangle-\left\langle z_{1}\right\rangle$. This difference occurs in the all quarters in the graphene ring. It is supposed that the initial pseudo spin current is parallel to the $x$ direction. When carriers pass the first quarter in graphene large ring, the pseudo spin current is the $y$ direction, in this condition, the initial state for $z_{2}$ is $z_{1}$, so $\delta\langle z\rangle=$ $-\left\langle\frac{e \hbar E_{x}}{\Delta m \omega_{2}} \sin \left(\frac{\omega_{2} t}{2}\right)\left(\sigma_{y} \cos \left(\frac{\omega_{2} t}{2}\right)+\sigma_{x} \sin \left(\frac{\omega_{2} t}{2}\right)\right)\right\rangle$ and in the end of second quarter the pseudo spin current go back along the $x$ direction again and this rotation for the carriers keeps continuing in this manner. Consequently, the $z_{1}, z_{2}, \delta z$ and spin force can vary with the number of charges in the central ring and geraphene gap. The main result of this paper is $\delta z$ and the Zitterbewegung component which are identical and both of them can be controlled by varying the energy gap.

\section{Conclusion}

Due to the similarity of the spin force and Lorentz force, the spin force plays a similar role in the formation of the charge Hall effect created by the spin current and the spin Hall effect driven by the charge current in an effective magnetic field. We obtained spin force on pseudo spin current and the positions with an effective magnetic field which create an effective precessional frequency. And also, we shown, the spin force can effect on spin of electrons in the rings of graphene and change frequently them in around the central ring. Finally, the graphene rings can be used as a memory or logical system based on pseudo spin magnetism which its performance is due to spin force, also the Zitterbewegung component can be controlled by varying graphnen's energy gap and electric field duo to charged nanodat at the central graphene ring.

\section{REFERENCES}

[1] K.S. Novoselov, A.K. Geim, S.V. Morozov, D. Jiang, Y. Zhang, S.V. Dubonos, I.V. Grigorieva, A.A. Firsov, Science 306, 666 (2004).

[2] A.K. Geim, K.S. Novoselov, Nature Mater. 6, 183 (2007).

[3] A.H. Castro Neto, F. Guinea, N.M.R. Peres, K.S. Novoselov, A.K. Geim, arXiv:0709.1163 [cond mat.other].

[4] P.R. Wallace, Phys. Rev. 71, 622 (1947).

[5] G.W. Semenoff, Phys. Rev. Lett. 53, 2449 (1984).

[6] D.P. Di Vincenzo, E.J. Mele, Phys. Rev. B 29, 1685 (1984). 
[7] S.G. Tan, M.B.A. Jalil, D.E. Koh, H.K. Lee, Y.H. Wu, arXiv:0806.1568 [cond-mat].

[8] S. Datta and B. Das, Appl. Phys. Lett. 56, 665 (1990)

[9] M.I. Dyakonov and V.I. Perel, JETP Lett. 13, 467 (1971); J.E. Hirsch, Phys. Rev. Lett. 83, 1834 (1999); J. Sinova et al, ibid. 92, 126603 (2004); S. Murakami et al, Science 301, 1348 (2003)

[10] Y-J. Lin, K. Jimenez-Garcia, and I. B. Spielman. Nature 471, no. 7336 (2011): 83-86.

[11] S. Datta, B. Das, Appl. Phys. Lett. 46, 665 (1989).

[12] R. Winkler, SpinOrbit Coupling Effects in Two-Dimensional Electron and Hole Systems, Springer, Berlin (2003).

[13] S.Q. Shen, Phys. Rev. Lett. 95, 187203 (2005).

[14] S.Q. Shen, Phys. Rev. Lett. 78, 1335 (2004).

[15] The Hall Effect and its Applications, edited by C. L. Chien and C. R. Westgate (Plenum, New York, 1980)

[16] E. McCann, , and V. I. Falko. Phys. Rev. Lett 96, no. 8 (2006): 086805.

[17] S. Murakami, N. Nagaosa and S.C. Zhang, Science 301,1348 (2003), J. Sinova et al., Phys. Rev. Lett. 92 , 126603 (2004).

[18] J. Anandan, Phys. Lett. A 138, 347 (1989); R. C. Casella and S. A. Werner, Phys. Rev. Lett. 69, 1625; A. V. Balatsky and B. L. Altshuler, Phys. Rev. Lett. 70, 1678 (1993); C. M. Ryu, Phys. Rev. Lett. 76, 968 (1996).

[19] D. Gyula, and J. Cserti, Physical Review B 81.12 (2010): 121417.

[20] J. Schliemann, D. Loss, and R. M. Westervelt, Phys. Rev. Lett. 94, 206801 (2005).

[21] H. Feshbach and F. Villars, Rev. Mod. Phys. 30, 24 (1958).

[22] A. O. Barut and A. J. Bracken, Phys. Rev. D 23, 2454 (1981).

[23] A. O. Barut and W. Thacker, Phys. Rev. D 31, 1386 (1985).

[24] F. Cannata and L. Ferrari, Phys. Rev. B 44, 8599 (1991).

[25] P. Krekora, Q. Su, and R. Grobe, Phys. Rev. Lett. 93, 043004 (2004).

[26] J. Cserti, G. Dvid . Phys. Rev. B, 74(17), 172305 (2006).

[27] M. Mamoru, et al, Physical Review B 84.10, 104410 (2011).

[28] S.Y. Zhou, G. H. Gweon, A.V. Fedorov et al., Nature Materials 6, 770 (2007).

[29] V.M. Pereira, V.N. Kotov, and A.H. Castro Neto,narXiv:0803.4195v1 (cond-mat. Mes-hall).

[30] H. Min, G. Borghi, M. Polini, and A.H. MacDonald, Phys. Rev. B 77, 041407(R) (2008).

[31] W. Greiner, Relativistic Quantum Mechanics, 3rd ed., Springer, Berlin (2000).

[32] L.P. Rokhinson et al, Phys. Rev. Lett. 93, 146601 (2004) 